\title{
ERK1/2 activation mediated by the nutlin-3-induced mitochondrial translocation of p53
}

\author{
SUN-YOUNG LEE ${ }^{1}$, SEOK JOON SHIN ${ }^{2}$ and HO-SHIK KIM ${ }^{1}$ \\ Departments of ${ }^{1}$ Biochemistry and ${ }^{2}$ Internal Medicine, College of Medicine, \\ The Catholic University of Korea, Seoul 137-701, Republic of Korea
}

Received October 30, 2012; Accepted December 6, 2012

DOI: 10.3892/ijo.2013.1764

\begin{abstract}
Nutlin-3 is a small-molecule antagonist of murine double minute 2 (MDM2) that blocks its binding to p53, leading to an increase in p53 protein levels. The tumor suppressor p53 induces growth arrest or apoptosis in response to genotoxic stress. Along with its growth-suppressive effect, it has been reported that p53 stimulates the mitogen-activated protein kinase (MAPK) pathway via the upregulation of heparinbinding epidermal growth factor-like growth factor (HB-EGF), an epidermal growth factor receptor (EGFR) ligand, and discoidin domain receptor 1 (DDR1), a tyrosine kinase receptor, at the transcription level. In this study, we propose a novel mechanism involved in the p53-induced MAPK activation. Nutlin-3 induced the phosphorylation of EGFR, MAPK/ERK kinase (MEK)1/2 and extracellular signal-regulated kinase (ERK)1/2 in U2OS human osteosarcoma cells harboring wildtype $\mathrm{p53}$. This phosphorylation was completely inhibited by $\mathrm{p} 53$ siRNA, but not by pifithrin (PFT)- $\alpha$, an inhibitor of the transcriptional activity of p53. While the nutlin-3-induced EGFR phosphorylation was prevented by the inactivation of ERK1/2, the nutlin-3-induced MEK1/2-ERK1/2 phosphorylation was still observed in the cells in which EGFR phosphorylation was inhibited using EGFR siRNA and AG1478, an inhibitor
\end{abstract}

Correspondence to: Dr Ho-Shik Kim, Department of Biochemistry, College of Medicine, The Catholic University of Korea, 505 Banpodong, Socho-gu, Seoul 137-701, Republic of Korea

E-mail: hoshik@catholic.ac.kr

Abbreviations: DDR1, discoidin domain receptor 1; DUSP5, dualspecificity phosphatase 5; EGR-1, early growth response-1; H2DCF-DA, 2'7'-dichlorodihydrofluorescein diacetate; EGFR, epidermal growth factor receptor; ERK, extracellular signal-regulated kinase; HB-EGF, heparin-binding epidermal growth factor-like growth factor; MAPK, mitogen-activated protein kinase; MDM2, murine double minute 2; MEK, MAPK/ERK kinase; MKP1, MAPK phosphatase 1; PAC1, phosphatase of activated cells 1; PFT, pifithrin; ROS, reactive oxygen species; TEMPO, 2,2,6,6-tetramethyl-1-piperidinyloxy

Key words: apoptosis, tumor suppressor protein p53, murine double minute 2 protein, mitogen-activated protein kinase kinase $1 / 2$, extracellular signal-regulated kinase $1 / 2$ of EGFR tyrosine kinase. Of note, nutlin-3 caused the accumulation of mitochondrial reactive oxygen species (ROS) and this correlated with the mitochondrial translocation of $\mathrm{p} 53$. 2,2,6,6-Tetramethyl-1-piperidinyloxy (TEMPO), a ROS scavenger, prevented the phosphorylation of ERK1/2. PFT- $\mu$, which prevented the mitochondrial localization of p53, suppressed ERK1/2 phosphorylation, as well as ROS accumulation. Finally, we analyzed the effect of ERK1/2 activation on nutlin-3induced apoptosis. The knockdown of MEK1/2 and ERK1/2 activity using U0126, a MEK inhibitor, or siRNAs, resulted in the enhancement of nutlin-3-induced apoptosis. In addition, TEMPO and PFT- $\mu$ also promoted nutlin-3-induced apoptosis. Taking the above findings into account, it can be concluded that nutlin-3 activates ERK1/2 prior to EGFR phosphorylation via $\mathrm{ROS}$ generation following the mitochondrial translocation of p53 and that nutlin-3-induced ERK1/2 activation may play a role in protecting U2OS cells from p53-dependent apoptosis, constituting a negative feedback loop for p53-induced apoptosis.

\section{Introduction}

The p53 protein, a transcription factor which exerts significant tumor-suppressive activity, regulates the expression of various genes that induce different anti-carcinogenesis mechanisms $(1,2)$. These include cell cycle arrest, apoptosis, senescence and DNA repair in response to genotoxic stress, depending on the type and severity of stress and the cellular context. Since the activation of p53 can lead to irreversible cellular injury, p53 protein levels are tightly regulated. Under basal conditions, the $\mathrm{p} 53$ protein has a short half-life, due to its binding to the murine double minute (MDM)2 protein, an E3 ubiquitin ligase that targets it for $26 \mathrm{~S}$ proteasomal degradation (3). MDM2 binding also hinders the interaction between p53 and transcriptional co-activators, such as p300 and the CREB-binding protein (CBP), thus rendering p53 inactive as a transcription factor (4). Due to its central role in cell death and cell cycle arrest, the activation of p53 has become an attractive therapeutic approach. In fact, currently used anticancer treatments, including chemotherapy and radiotherapy, induce p53-dependent apoptosis in cancer cells with an intact p53 gene. These therapeutic methods induce genomic DNA damage, resulting in the activation of $\mathrm{p} 53$, which makes cancer cells with rapid DNA replication more susceptible to these treatments compared to non-transformed cells (5). However, 
these treatments may cause DNA damage to normal cells and, in fact, certain studies have reported the development of tumors, such as leukemia, bladder, kidney and breast cancers, following such anticancer treatments $(6,7)$. Therefore, small molecules such as MDM2 inhibitors, that induce p53 activation without causing DNA damage, may represent promising anticancer drugs, since they reduce the risk of secondary tumor development $(8,9)$. Nutlin-3, a cis-imidazoline analog, was the first selective MDM2 inhibitor to be discovered (10). The structure of nutlin-3 is similar to that of amino acids in the binding domain between 553 and MDM2. It binds to the p53-binding pocket of MDM2, thereby displacing p53 (from MDM2) and upregulating the p53 protein. As shown in previous studies using the murine xenograft model, the treatment of cancer cells with nutlin-3 triggers substantial p53-dependent cell cycle arrest and apoptosis in vitro and exhibits antitumor efficacy $(10,11)$. It is noteworthy that MDM2 inhibitors, including nultin-3, are not toxic to normal cells $(12,13)$.

The Ras-Raf-MEK1/2-ERK1/2 pathway, which is generally activated by growth factors, controls various cellular responses, such as proliferation, migration, differentiation and death $(14,15)$. Upon growth factor stimuli, Ras is activated via receptor tyrosine kinases (RTKs) and subsequently activates Raf, MAPK/ERK kinase (MEK) and extracellular signal-regulated kinase (ERK), leading to the activation of a number of transcription factors that orchestrate various cellular responses. The Ras-Raf-MEK-ERK signaling cascade has been demonstrated to functionally interact with the $\mathrm{p} 53$ pathway. For example, ERK1/2 is required for the phosphorylation of $\mathrm{p} 53$ and apoptosis induced by cisplatin, colcemid, resveratrol and benzo(a)pyrene in ovarian cancer, fibroblast, epidermal and hepatocellular carcinoma cells, respectively (16-19). However, it has also been reported that p53 is capable of modulating ERK1/2 activation, although its effects on ERK1/2 activity do not appear to be consistent. In human bladder cancer, osteosarcoma and colon cancer cells, p53 activates the Ras-RafMEK1/2-ERK1/2 pathway via the transcriptional induction of heparin-binding epidermal growth factor-like growth factor (HB-EGF) and discoidin domain receptor 1 (DDR1), to protect cancer cells against p53-dependent apoptosis (20-22). By contrast, p53 suppresses ERK1/2 activity by activating phosphatase genes, such as phosphatase of activated cells 1 (PAC1), dual-specificity phosphatase 5 (DUSP1) and mitogenactivated protein kinase (MAPK) phosphatase 1 (MKP1), which can dephosphorylate ERK1/2 (23-25). Since PAC1, DUSP1 and MKP1 can block the anti-apoptotic and cell cycle progression effects of ERK1/2, p53 induces apoptosis and cell cycle arrest via the upregulation of these phosphatase genes. ERK1/2 thus appears to act as an upstream activator of $\mathrm{p} 53$ phosphorylation or a downstream effector of p53 that counteracts p53-induced apoptosis, depending on the cell type and p53-activating stimuli, although the mechanisms involved remain to be elucidated.

Recent studies have reported that the combined treatment with nutlin-3 and a MEK inhibitor synergistically induces apoptosis in AML cells in which the MAPK pathway is aberrantly activated, and which overexpress MDM2 $(26,27)$. This synergistic activation of apoptosis occurred, in part, via the phosphorylation of FOXO3a, leading to an increase in pro-apoptotic genes, such as p53 upregulated modulator of apoptosis (PUMA) and BIM. These findings suggest that the growth-suppressive activity of nutlin-3 may also be functionally counteracted by the MAPK pathway. However, the correlation between nutlin-3-induced p53 and MAPK activity remains unclear. To address this issue, we analyzed MAPK activation and the underlying mechanisms in nutlin-3-treated U2OS cells, a human osteosarcoma cell line with established susceptibility to nutlin-3.

\section{Materials and methods}

Reagents. Nutlin-3 and U0126 were purchased from Tocris (Bristol, UK). Pifithrin (PFT)- $\alpha$ and $-\mu, 2^{\prime}, 7^{\prime}$-dichlorodihydrofluorescein diacetate (H2DCF-DA) and 2,2,6,6-tetramethyl-1piperidinyloxy (TEMPO) were obtained from SigmaAldrich Inc. (St. Louis, MO, USA). MitoSOX ${ }^{\mathrm{TM}}$ Red reagent was obtained from Invitrogen (Carlsbad, CA, USA).

Cell culture. U2OS human osteosarcoma cells were cultured in DMEM supplemented with $10 \%$ heat-inactivated fetal bovine serum (Hyclone, Logan, UT, USA), $100 \mathrm{U} / \mathrm{ml}$ penicillin (Hyclone) and glutamate (Invitrogen) at $37^{\circ} \mathrm{C}$ with $5 \% \mathrm{CO}_{2}$.

Antibodies. Mouse antibodies against phospho-p42/44, p53 and GAPDH were purchased from Santa Cruz Biotechnology (Santa Cruz, CA, USA). Rabbit anti-p42/44 and mouse anti-phospho-MEK1/2 were obtained from Cell Signaling Technology (Boston, MA, USA). Rabbit anti-MEK1 and anti-MEK2 were obtained from Epitomics (Burlingame, CA, USA). Secondary antibodies against rabbit or mouse IgG were obtained from Sigma-Aldrich Inc.

Transfection of small interfering RNAs (siRNAs). p53 siRNA was obtained from Santa Cruz Biotechnology and siRNAs against MEK1, MEK2, ERK1, ERK2 and epidermal growth factor receptor (EGFR) were purchased from Sigma-Aldrich Inc. siRNA transfection was carried out using Lipofectamine $^{\mathrm{TM}}$ RNAiMAX (Invitrogen), following the manufacturer's instructions.

Immunoblot analysis. Cells treated as indicated in figures were lysed with RIPA buffer (Cell Signaling Technology) containing protease inhibitor cocktail (Roche, Basel, Switzerland) or with 1X SDS sample buffer. Equal amounts of protein were separated by SDS-polyacrylamide gel electrophoresis and transferred onto nitrocellulose membranes (Millipore, Billerica, MA, USA). The membranes were incubated in 5\% non-fat dried milk in TBS-T (Tris-buffered saline with $0.05 \%$ Tween-20) containing primary antibodies and then incubated with secondary antibodies, followed by chemiluminescence (ECL, GE Healthcare, Buckinghamshire, UK)-based detection.

Quantitative real-time $R T-P C R(q R T-P C R)$. Total RNA was isolated using RNAiso Plus (Takara Bio Inc., Shiga, Japan) and first-strand cDNA was generated using the PrimeScript ${ }^{\mathrm{TM}} \mathrm{RT}$ reagent kit (Takara Bio Inc.). Quantitative real-time RT-PCR was performed using SYBR Premix Ex Taq (Takara Bio Inc.) and the ABI 7300 Real-Time PCR system (Applied Biosystems, Carlsbad, CA, USA). All reactions were performed following the manufacturer's instructions. Relative quantification was 
performed using the $\triangle \triangle \mathrm{Ct}$ method with $G A P D H$ as the endogenous control (28).

Apoptosis assay. Apoptosis was assessed by measuring the number of hypodiploidic cells and the translocation of phosphatidylserine using flow cytometry (FACSCalibur; BD Biosciences, San Jose, CA, USA). To measure the number of hypodiploidic cells, cells treated as indicated in Fig. 5 were harvested, fixed in ice-cold $70 \%$ ethanol for $2 \mathrm{~h}$ and then stained with propidium iodide (PI, $0.2 \mathrm{mg} / \mathrm{ml}$ ). The stained cells were analyzed on a FACSCalibur and cell cycle distribution was calculated by CellQuest and ModFit software (BD Biosciences). For determination of phosphatidylserine translocation, cells were stained with PI and Annexin V using the ApoScan kit (BioBud, Gyunggido, Korea), followed by flow cytometry analysis as described previously (29).

Isolation of cytosolic and mitochondrial fractions. Cells treated as indicated in Fig. 4 were resuspended in ice-cold IBc buffer (0.1 M Tris-MOPS, 0.5 M EGTA/Tris, $1 \mathrm{M}$ sucrose, $\mathrm{pH}$ 7.4) containing a protease inhibitor cocktail (Roche). The cells were homogenized using dounce homogenizers and centrifuged at $6,000 \mathrm{x} \mathrm{g}$ for $10 \mathrm{~min}$ at $4^{\circ} \mathrm{C}$ to remove unlysed cells and nuclei. The supernatant was collected and centrifuged at 7,000 x g for $10 \mathrm{~min}$ at $4^{\circ} \mathrm{C}$. The clear fraction was saved as the cytosolic fraction and the pellet was resuspended in $\mathrm{IBc}$ buffer, washed again and centrifuged at 10,000 x g for $10 \mathrm{~min}$ at $4^{\circ} \mathrm{C}$. The pellet was resuspended in RIPA buffer and used as the mitochondrial fraction.

Measurement of reactive oxygen species (ROS). To measure intracellular ROS levels and mitochondrial ROS, cells were incubated in the presence of $20 \mu \mathrm{M} \mathrm{H} 2 \mathrm{DCF}-\mathrm{DA}$ dye and $5 \mu \mathrm{M}$ MitoSOX Red reagent working solution (Invitrogen), respectively, for $15 \mathrm{~min}$. After the cells were washed twice in pre-warmed PBS, they were examined under a fluorescence inverted microscope (Olympus IX71, Tokyo, Japan) and the intensity of the fluorescence was measured by flow cytometry.

\section{Results}

Nutlin-3 induces the phosphorylation of MEK1/2-ERK1/2 in a p53-dependent manner. To determine whether the upregulation of $\mathrm{p} 53$ by nutlin- 3 induces the phosphorylation of ERK1/2, we treated U2OS cells with $20 \mu \mathrm{M}$ nutlin-3, treatment conditions that have been reported to induce apoptosis in this cell line (10). As shown in Fig. 1A, treatment with nutlin-3 resulted in the accumulation of p53 protein and p21WAF1 mRNA in a time-dependent manner, indicating that p53 was activated by nutlin-3. Along with p53 activation, the phosphorylation of ERK1/2 and MEK1/2 was induced by nutlin-3 as well, which was also dependent on the incubation time (Fig. 1A). The activation of p53 and the phosphorylation of MEK1/2-ERK1/2 commenced after $6 \mathrm{~h}$ of nutlin-3 treatment and were still observed after $48 \mathrm{~h}$ of treatment. Therefore, to elucidate the mechanism behind this phenomenon, we used $20 \mu \mathrm{M}$ nutlin-3 and a 24-h incubation time for the following experiments. We then determined whether the nutlin-3-induced MEK1/2 and ERK1/2 phosphorylation was the direct effect of p53 activation. As shown in Fig. 1B, the downregulation of p53
A
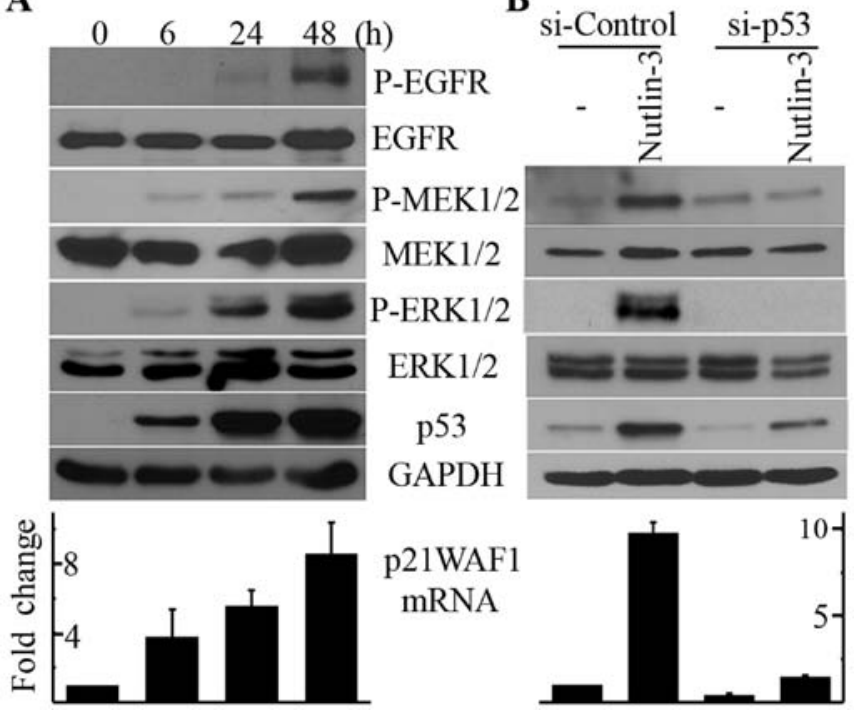

Figure 1. Phosphorylation of MEK1/2-ERK1/2 by nutlin-3 in a p53-dependent manner. (A) Time-dependent induction of MEK1/2-ERK1/2 phoshorylation by nutlin-3. U2OS cells were treated with $20 \mu \mathrm{M}$ nutlin-3. At the indicated time points after the treatment, total cell lysates and total cellular RNAs were prepared and subjected to immunoblot analyses against the indicated antibodies (upper panel) and quantitative real-time RT-PCR (qRT-PCR) against p21WAF1 (lower panel), respectively, as described in Materials and methods. (B) Effect of p53 siRNA on nutlin-3-induced MEK1/2-ERK1/2 phosphorylation. U2OS cells transfected with scrambled siRNA (si-Control) as the negative control or p53 siRNA (si-p53) for $48 \mathrm{~h}$ were treated with the vehicle or $20 \mu \mathrm{M}$ nutlin-3. After $24 \mathrm{~h}$, whole cell lysates and total cellular RNAs were extracted and subjected to immunoblot analyses against the indicated antibodies (upper panel) and qRT-PCR against p53 and p21WAF1 (lower panel) as described in Materials and methods. GAPDH was used as the loading control of proteins for immunoblot analyses and as an internal reference for real-time qRT-PCR. P-EGFR, phospho-EGFR (S1173); P-MEK1/2, phospho-MEK1/2 (S217/221); P-ERK1/2, phospho-ERK1/2 (T202/Y204).

by RNA interference almost completely inhibited the nutlin3-induced phosphorylation of MEK1/2 and ERK1/2 as well as the increase in p21WAF1 mRNA levels. Therefore, it can be concluded that nutlin-3 induces MEK1/2-ERK1/2 phosphorylation by activating p53 in U2OS cells.

Nutlin-3-induced MEK1/2-ERK1/2 activation is not dependent on EGFR activation or the transcriptional activity of p53. Since p53 has been reported to induce EGFR phosphorylation via the upregulation of EGFR ligands such as HB-EGF (21) and it has also been argued that EGFR ligands can be induced downstream of ERK1/2 via early growth response-1 (EGR-1) (30), we examined whether nutlin-3 induced the activation of EGFR and whether this was responsible for the phosphorylation of MEK1/2-ERK1/2. Nutlin-3 induced the phosphorylation of EGFR as well as that of MEK1/2-ERK1/2 in a time-dependent manner (Fig. 1A). However, when EGFR phosphorylation was prevented by EGFR siRNA and AG1478, an EGFR kinase inhibitor, the phosphorylation of MEK1/2-ERK1/2 was not affected (Fig. 2A; data not shown). By contrast, EGFR phosphorylation was almost completely prevented by ERK1/2 siRNA and U0126, a MEK inhibitor (Fig. 2B and C), suggesting that ERK1/2, when activated by nutlin-3, induces the phosphorylation of EGFR and that the transcriptional activity of p53 may not be involved in this 

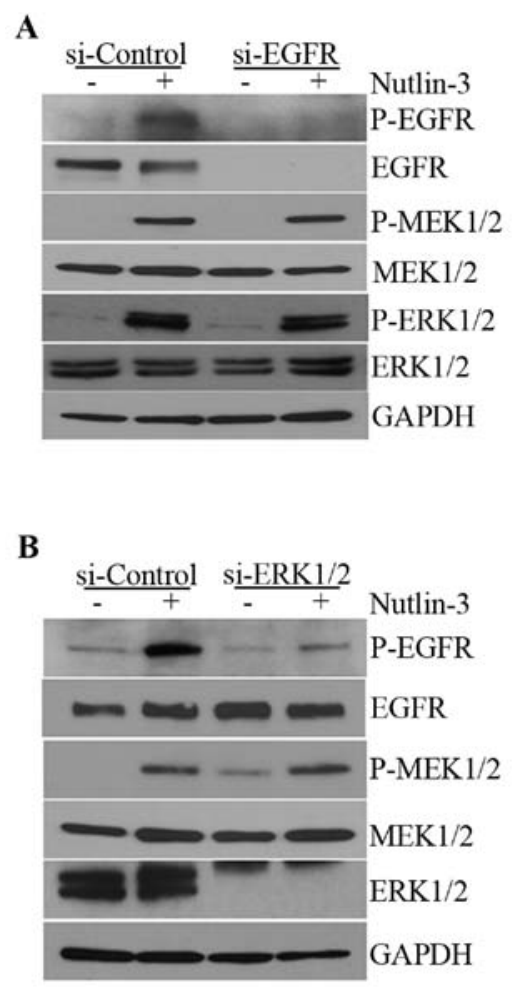

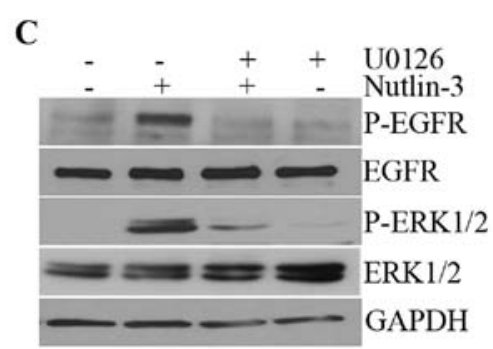

D

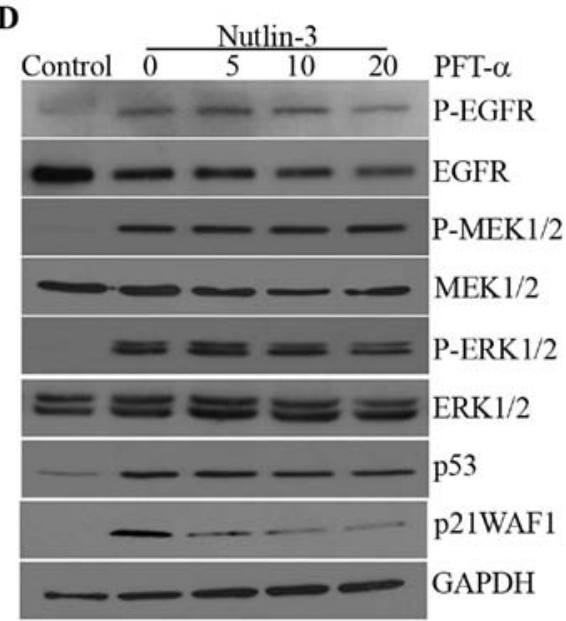

Figure 2. Nutlin-3-induced MEK1/2-ERK1/2 activation is not dependent on EGFR activation. (A) Effect of EGFR siRNA (si-EGFR) on the phosphorylation of MEK1/2-ERK1/2. U2OS cells transfected with si-EGFR for $48 \mathrm{~h}$ were cultured in the presence of the vehicle or $20 \mu \mathrm{M}$ nutlin-3. After $24 \mathrm{~h}$ of treatment, cell lysates were subjected to immunoblot analyses. (B) Effect of ERK1/2 siRNA (si-ERK1/2) on the phosphorylation of EGFR. U2OS cells transfected with si-ERK1/2 for $48 \mathrm{~h}$ were treated with the vehicle or $20 \mu \mathrm{M}$ nutlin-3. After $24 \mathrm{~h}$ of treatment, cell lysates were subjected to immunoblot analyses. (C) Effect of U0126 on the phosphorylation of EGFR. U2OS cells treated with the vehicle or $20 \mu \mathrm{M}$ U0126 for $1 \mathrm{~h}$ were incubated in the presence of the vehicle or $20 \mu \mathrm{M}$ nutlin-3 for a further $24 \mathrm{~h}$. Cell lysates were then subjected to immunoblot analyses. (D) U2OS cells treated with indicated concentrations of PFT- $\alpha$ for $1 \mathrm{~h}$, were treated with the vehicle or $20 \mu \mathrm{M}$ nutlin-3. After $24 \mathrm{~h}$, cell lysates were subjected to immunoblot analyses. GAPDH was used as the loading control for each sample. P-EGFR, phospho-EGFR (S1173); P-MEK1/2, phospho-MEK1/2 (S217/221); P-ERK1/2, phospho-ERK1/2 (T202/Y204).

MEK1/2-ERK1/2 phosphorylation. Whereas an increase in p21WAF1 mRNA levels was almost completely inhibited by PFT- $\alpha$, an inhibitor of the transcriptional activity of p53, the phosphorylation of MEK1/2-ERK1/2 was not altered (Fig. 2D). This suggests that nutlin-3 may induce MEK1/2-ERK1/2 and subsequently, EGFR phosphorylation through a mechanism that is independent of the transcriptional activity of p53.

Involvement of ROS in the activation of MEK1/2-ERK1/2 by nutlin-3. Since ROS have been reported to mediate the activation of ERK1/2 in various cells $(31,32)$ and p53 modulates the production of ROS (33), we examined the involvement of ROS in nutlin-3-induced ERK1/2 phosphorylation. As expected, ROS accumulated in the nutlin-3-treated cells (Fig. 3A) and this accumulation was prevented by a pre-treatment with TEMPO, a ROS scavenger (Fig. 3B). Parallel to its effect on ROS accumulation, TEMPO almost completely inhibited the phosphorylation of ERK1/2 and EGFR, but the MEK1/2 phosphorylation induced by nutlin-3 was only slightly inhibited (Fig. 3C). These findings demonstrated that the nutlin-3 induced ROS generation and accumulation of ROS contributed to the phosphorylation of ERK1/2.

Nutlin-3-induced ROS generation and MEK1/2-ERK1/2 activation are dependent on the mitochondrial translocation of p53. Since PFT- $\alpha$ had no effect on the nutlin-3-induced
MEK1/2-ERK1/2 phosphorylation (Fig. 2D), ROS accumulation caused by nutlin-3 may be independent of the transcriptional activity of p53. p53 has been reported to translocate to the mitochondria $(34,35)$, the main source of ROS, prompting us to examine the correlation between ROS accumulation and the mitochondrial translocation of p53 in nutlin-3-treated cells. For this purpose, we used MitoSOX, a reagent that captures mitochondrial ROS and immunoblot analyses of subcellular fractions. As shown in Fig. 4A, the nutlin-3-treated cells were stained with MitoSOX. The results showed that accumulating ROS were generated from the mitochondria and that nutlin-3 caused the mitochondrial translocation of p53. Both this mitochondrial p53 translocation and ROS generation were prevented by PFT- $\mu$, an inhibitor of the mitochondrial translocation of p53 (Fig. 4B). The staining of PFT- $\mu$-pre-treated cells with H2DCF-DA demonstrated that PFT- $\mu$ completely prevented ROS accumulation, suggesting that nutlin-3-induced ROS originated solely in mitochondria (Fig. 4B, right panel). It should be noted that, while the phosphorylation of ERK1/2 and EGFR by nutlin-3 was almost completely inhibited by PFT- $\mu$ pre-treatment, the phosphorylation of MEK1/2 was not inhibited, but rather potentiated by PFT- $\mu$. These results demonstrate that nutlin-3 induces the mitochondrial translocation of p53, which generates ROS in the mitochondria and that mitochondrial ROS are involved in the phosphorylation of ERK1/2 but not that of MEK1/2. 
A

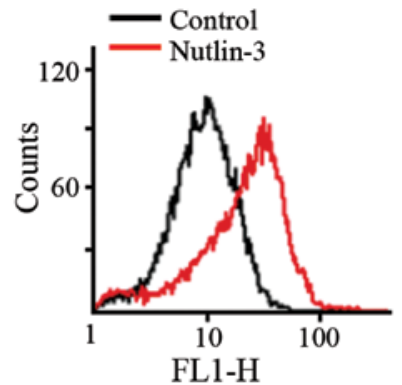

C

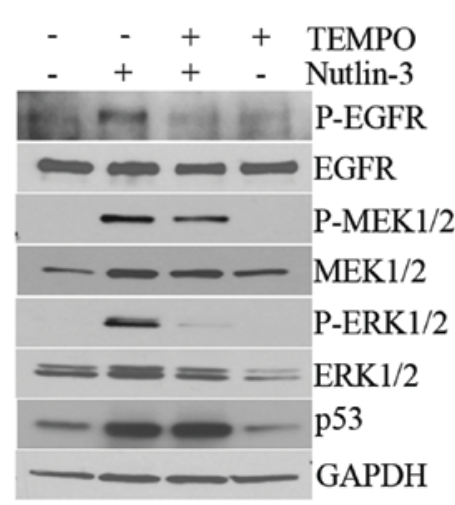

B
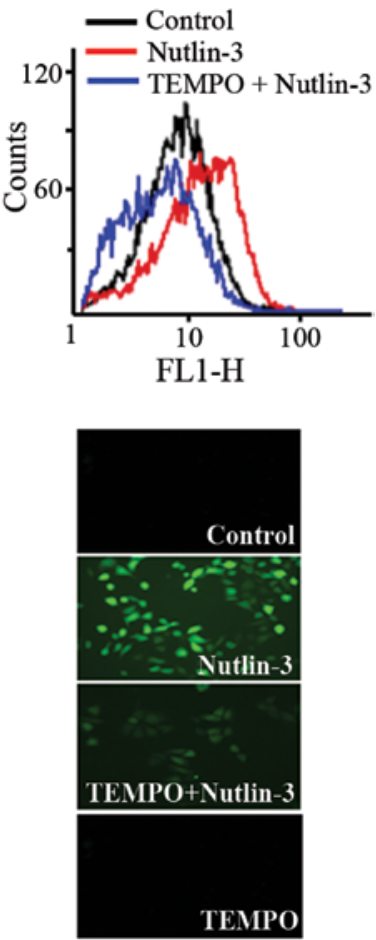

Figure 3. The involvement of ROS in the nutlin-3-induced activation of MEK1/2-ERK1/2. (A) ROS accumulation following nutlin-3 treatment. U2OS cells treated with the vehicle or $20 \mu \mathrm{M}$ nutlin-3 were stained by H2DCF-DA for $15 \mathrm{~min}$. The intensities of intracellular DCF were then analyzed on a FACSCalibur as described in Materials and methods. (B) Effect of TEMPO on nutlin-3-induced ROS accumulation. U2OS cells pre-treated with the vehicle or $2 \mathrm{mM}$ TEMPO were incubated in the absence or presence of $20 \mu \mathrm{M}$ nutlin-3 for $24 \mathrm{~h}$. Cells were then stained with H2DCF-DA and analyzed on a FACSCalibur. (C) Effect of TEMPO on nutlin3-induced MEK1/2-ERK1/2 phosphorylation. U2OS cells treated with the vehicle or $2 \mathrm{mM}$ TEMPO were incubated in the absence or presence of $20 \mu \mathrm{M}$ nutlin-3 for $24 \mathrm{~h}$. Cells were then subjected to immunoblot analyses against indicated proteins (left panel) and stained with H2DCF-DA (right panel). GAPDH was the protein loading control. P-EGFR, phospho-EGFR (S1173); P-MEK1/2, phospho-MEK1/2 (S217/221); P-ERK1/2, phospho-ERK1/2 (T202/Y204).

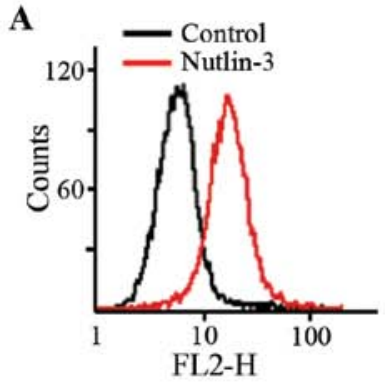

B

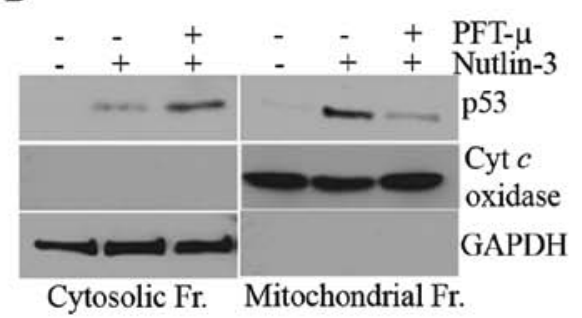

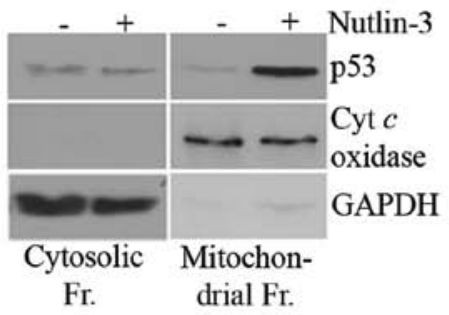

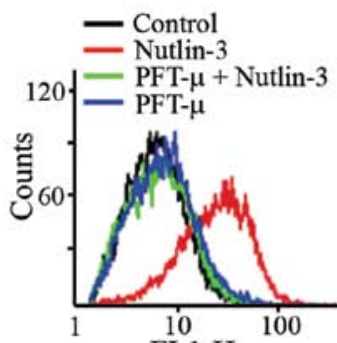

C

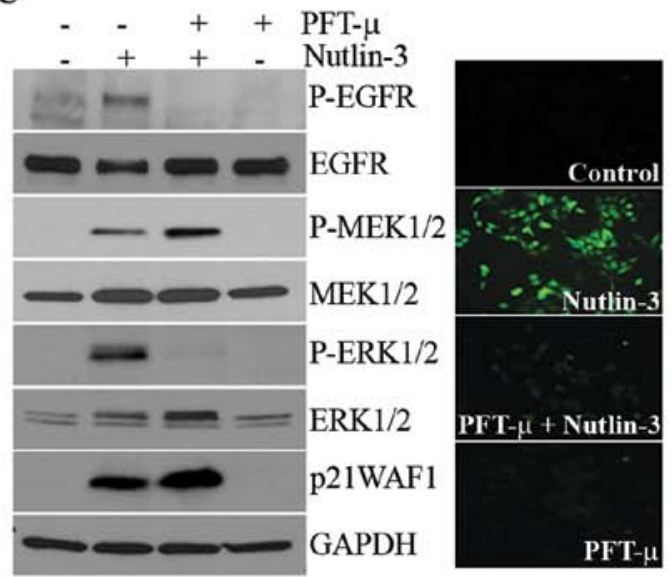

Figure 4. Nutlin-3-induced ROS generation and MEK1/2-ERK1/2 activation are dependent on the mitochondrial translocation of p53. (A) Mitochondrial translocation of p53 and mitochondrial ROS generation by nutlin-3. U2OS cells were treated with the vehicle or $20 \mu \mathrm{M}$ nutlin-3. After 24 h, cells were stained with MitoSOX $^{\mathrm{TM}}$ for $15 \mathrm{~min}$ and analyzed on a FACSCalibur (left panel) and subjected to subcellular fractionation into cytosolic (cytosolic Fr.) and mitochondrial (mitochondrial Fr.) fractions for immunoblot analyses against indicated proteins (right panel). Cytochrome $c$ (Cyt $c$ ) oxidase and GAPDH were used as mitochondrial and cytosolic protein loading controls, respectively. (B) Effect of PFT- $\mu$ on the mitochondrial translocation of p53 and ROS accumulation. U2OS cells were treated with $10 \mu \mathrm{M}$ PFT- $\mu$ for $1 \mathrm{~h}$ and incubated with the vehicle or $20 \mu \mathrm{M}$ nutlin-3. After $24 \mathrm{~h}$, cytosolic and mitochondrial fractions were subjected to immunoblot analyses (left panel) and cells were stained with H2DCF-DA, followed by FACSCalibur analysis (right panel). (C) Effect of PFT- $\mu$ on nutlin-3-induced MEK1/2-ERK1/2 phosphorylation. U2OS cells were treated with $10 \mu \mathrm{M}$ PFT- $\mu$ for $1 \mathrm{~h}$ and incubated in the absence or presence of $20 \mu \mathrm{M}$ nutlin-3. After $24 \mathrm{~h}$, cell lysates were subjected to immunoblot analyses against indicated proteins (left panel) and stained with MitoSOX for 15 min, followed by fluorescence microscopic observation (right panel). GAPDH was the protein loading control. P-EGFR, phospho-EGFR (S1173); P-MEK1/2, phospho-MEK1/2 (S217/221); P-ERK1/2, phospho-ERK1/2 (T202/Y204). 
A

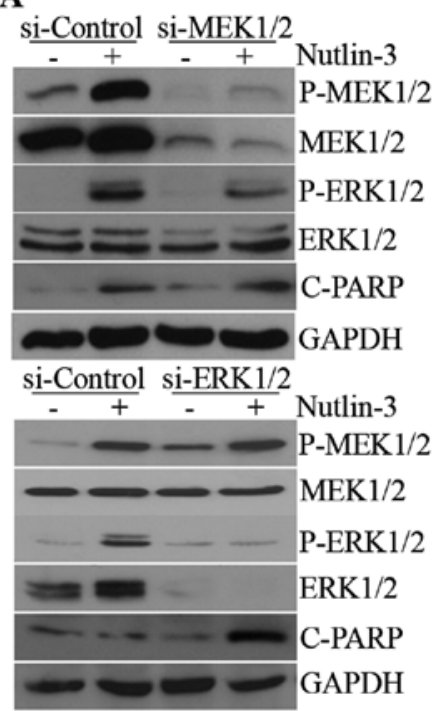

B

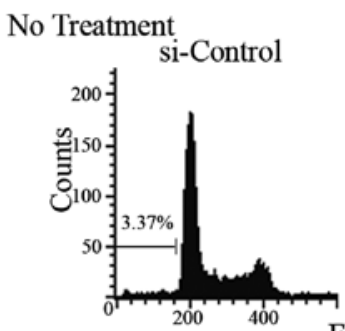

Nutlin-3 Treatment

FL2-A

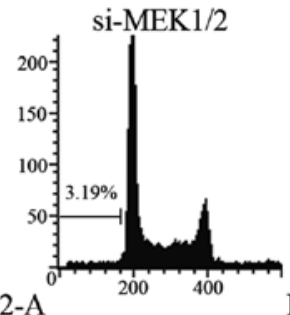

FL2-A
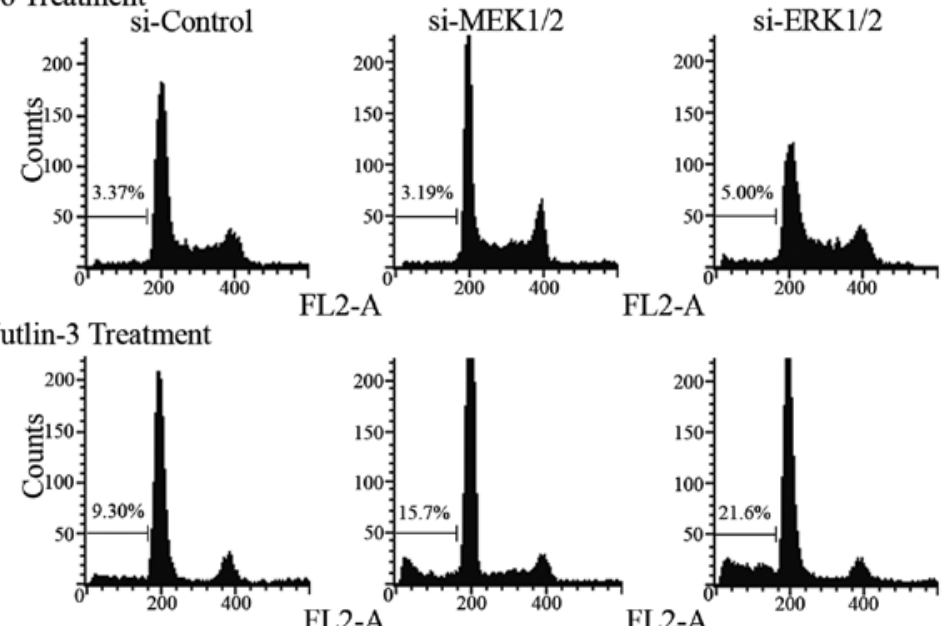

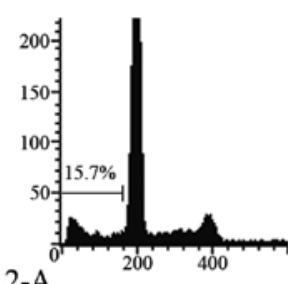

FL2-A

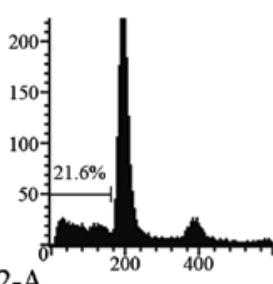

C

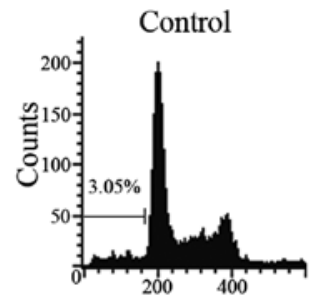

FL2-A

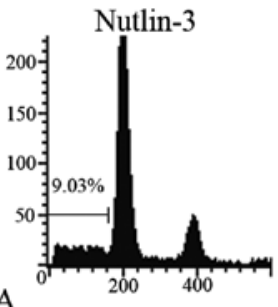

FL2-A

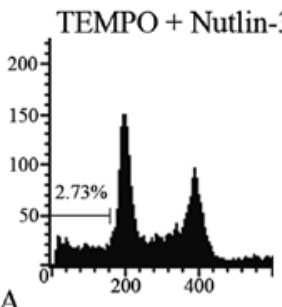

FL2-A

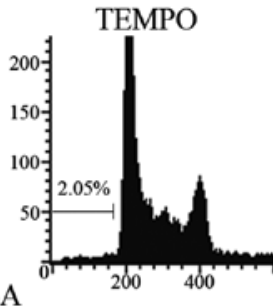

D

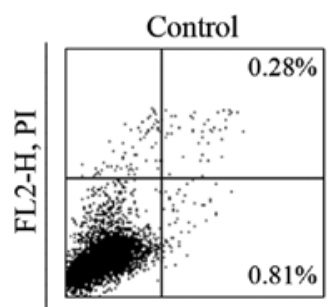

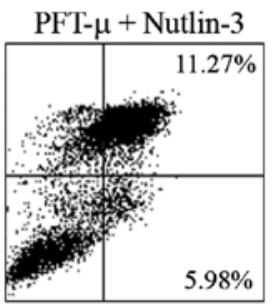

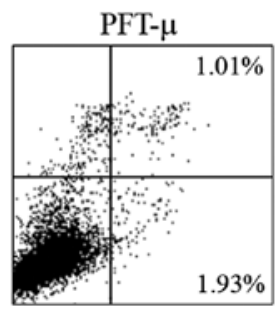

FL1-H, Annexin-V

Figure 5. Effect of MEK1/2-ERK1/2 inhibition on nutlin-3-induced apoptosis. (A and B) Effect of MEK1/2 siRNA and ERK1/2 siRNA on nutlin-3-induced apoptosis. U2OS cells transfected with scramble siRNA (si-Control) as the negative control, MEK1/2 siRNA (si-MEK1/2) or ERK1/2 siRNA (si-ERK1/2) for $48 \mathrm{~h}$ were treated with the vehicle or $20 \mu \mathrm{M}$ nutlin-3. After $24 \mathrm{~h}$, cells were subjected to immunoblot analyses (A) and stained with propidium iodide, followed by cell cycle analysis on a FACSCalibur (B). P-MEK1/2, phospho-MEK1/2 (S217/221); P-ERK1/2, phospho-ERK1/2 (T202/Y204); C-PARP, cleaved poly(ADP-ribose) polymerase (p25). (C) Effect of TEMPO on nutlin-3-induced apoptosis. U2OS cells were pre-treated with $2 \mathrm{mM}$ TEMPO for $1 \mathrm{~h}$ and then treated with vehicle or $20 \mu \mathrm{M}$ nutlin-3. After $24 \mathrm{~h}$ of nutlin-3 treatment, cells were stained with propidium iodide, followed by cell cycle analysis on a FACSCalibur. (D) Effect of PFT- $\mu$ on nutlin-3-induced apoptosis. U2OS cells were pre-treated with vehicle or $10 \mu \mathrm{M}$ PFT- $\mu$ and incubated in the absence or presence of $20 \mu \mathrm{M}$ nutlin-3 for a further $24 \mathrm{~h}$. Cells were then stained with both Annexin V and propidium iodide, followed by flow cytometric analysis on a FACSCalibur as described in Materials and methods.

Effect of ERK1/2 inhibition on nutlin-3-induced cell death. We then attempted to determine the role of MEK1/2-ERK1/2 phosphorylation on nutlin-3-induced cell death. When the phosphorylation of MEK1/2 and ERK1/2 was reduced by the knockdown of MEK1/2 and ERK1/2 mediated by the respective siRNAs (Fig. 5A), nutlin-3-induced apoptotic features, such as the cleavage of poly(ADP-ribose) polymerase-1 (PARP-1) and the accumulation of hypodiploidic cells, were augmented (Fig. 5A and B). The effect of the inhibition of ERK1/2 phosphorylation by U0126, a chemical inhibitor of MEK1/2, was consistent with that of siRNAs against MEK1/2 and ERK1/2 (data not shown). Furthermore, pre-treatment with TEMPO and PFT- $\mu$, decreased ERK1/2 phosphorylation, and increased nutlin-3-induced apoptosis (Fig. 5C and D). These results demonstrate that the MEK1/2-ERK1/2 activation suppresses nutlin-3-induced apoptosis, suggesting that it may constitute a negative feedback loop against nutlin-3-induced apoptosis in U2OS cells.

\section{Discussion}

In this study, we report that the $\mathrm{p} 53$ protein, when stabilized by nutlin-3 treatment, causes the activation of the MEK1/2-ERK1/2 pathway that functions as a survival pathway, thereby constituting a negative feedback loop against p53-induced apoptosis. The findings of the present study are summarized in the form of a schematic diagram, presented in Fig. 6. Since MEK1/2-ERK1/2 phosphorylation occurs downstream of EGFR activation and 


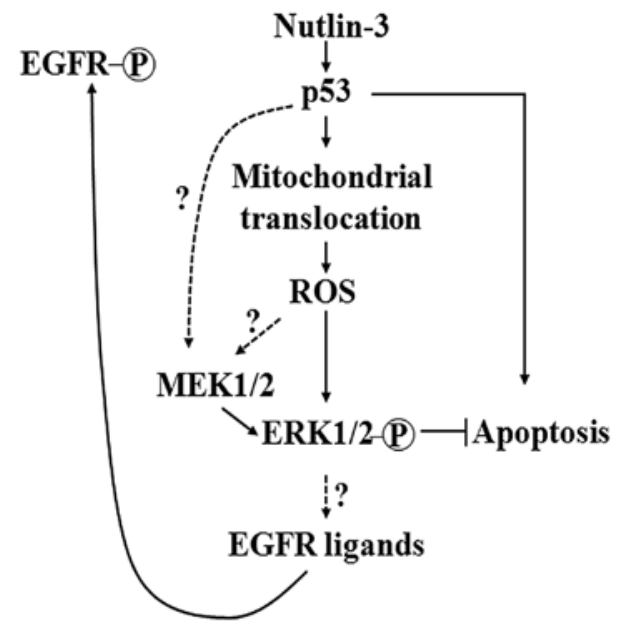

Figure 6. Schematic diagram of nutlin-3-induced ERK1/2 phosphorylation via mitochondrial p53 in U2OS cells. Nutlin-3-activated p53 moves to the mitochondria and induces ROS accumulation. The phosphorylation of ERK1/2 is induced by both ROS generated from the mitochondria and MEK1/2, the activation mechanism of which is not identified here. Activated ERK1/2 induces the phosphorylation of EGFR, possibly by the upregulation of EGFR ligands Consequently, MEK1/2-ERK1/2 acts as a negative feedback loop suppressing nutlin-3-induced apoptosis.

p53 activates EGFR by increasing the expression of HB-EGF at the transcription level (21), we hypothesized that the sequence of events in the nutlin-3-treated cells would be an increase of p53, followed by an increase of EGFR ligands, EGFR activation and finally MEK1/2-ERK1/2 phosphorylation. However, while the phosphorylation of MEK1/2-ERK1/2 was unaffected by the EGFR inhibition and PFT- $\alpha$, the phosphorylation of EGFR was completely prevented by the inhibition of ERK $1 / 2$, indicating that ERK1/2 activation occurs upstream of EGFR activation (Fig. 2). In accordance with this hypothesis, nutlin-3 induced the transcription of EGFR ligands such as amphiregulin, epiregulin and $H B-E G F$ and this induction was suppressed by ERK1/2 inhibitors but not by PFT- $\alpha$ (data not shown), leading to the assumption that the MEK1/2-ERK1/2 pathway may be activated by nutlin-3-induced p53 in a transcriptionindependent manner prior to the phosphorylation of EGFR. Mitochondrial ROS generation was increased during the nutlin-3 treatment and ROS accumulation closely correlated with the mitochondrial translocation of $\mathrm{p} 53$. While MEK1/2 activation was not inhibited or slightly inhibited by PFT- $\mu$ or ROS scavengers, respectively, ERK1/2 appeared to be almost completely inhibited by these compounds as well as by MEK inhibition (Fig. 3). Collectively, these findings led us to conjecture that the mitochondrial translocation of p53 and MEK1/2 activation are central events in nutlin-3-induced ERK1/2 activation and that the nutlin-3-induced phosphorylation of ERK1/2 may be induced by two pathways which act independently or mutually cooperatively: the sequence of events of one pathway is the increase of p53, the mitochondrial translocation of p53, followed by the mitochondrial generation of ROS and ERK1/2 phosphorylation, while that of the other pathway is the activation of MEK1/2 by $\mathrm{p} 53$, followed by ERK1/2 phosphorylation (Fig. 6).

In a number of studies, p53 has been shown to migrate to the mitochondria where it interacts with $\mathrm{Bcl}-\mathrm{xL}$, thereby activating pro-apoptotic $\mathrm{BAX}$ and $\mathrm{BAK}$ proteins to induce apoptosis $(34,35)$. Palacios et al and Talos et al induced apoptosis using p53-overexpressing constructs that target the mitochondria, such as Lp53WT and Lp53CBT, emphasizing the importance of the mitochondrial translocation of p53 in p53-induced apoptosis $(36,37)$. Moreover, it was recently reported that nutlin-3 also induced apoptosis via the mitochondrial translocation of p53 (38). Based on these reports, we hypothesized that the targeting of mitochondrial p53 using these constructs might induce ROS generation in the U2OS cell line. However, although these constructs appeared to move to the mitochondria, they failed to induce ROS generation and ERK1/2 phosphorylation (data not shown). It was therefore concluded that the mitochondrial translocation of p53 is necessary but not sufficient for ROS accumulation and ERK1/2 activation in U2OS cells. This conclusion led to the conjecture that two central pathways involving ROS accumulation and MEK1/2 activation may cooperate rather than act independently to activate ERK1/2 in nutlin-3-treated U2OS cells. The discrepancy between the effects of nutlin-3-induced p53 mitochondrial translocation and the p53-overexpressing constructs that target the mitochondria on ROS generation, suggests that there are other critical factors, such as the precise location and binding partners of p53 in mitochondria, that determine the effect of the mitochondrial translocation of p53 on apoptosis, ROS generation and MAPK activation.

The exact mechanism by which the nutlin-3-induced mitochondrial translocation of $\mathrm{p} 53$ regulates ROS accumulation remains to be clarified. Similar to the mechanism of apoptosis induction, p53 has been reported to induce ROS accumulation by both transcription-dependent and -independent mechanisms. Elevated p53 levels caused by genotoxic stress induce the transcription of pro-oxidant genes, such as NQO1, POX, BAX and PUMA, and suppress anti-oxidant genes, including MnSOD, thereby shifting the balance between ROS-generating and ROS-scavenging capacities in favor of ROS accumulation (33). Furthermore, when in the mitochondria, p53 increases ROS by binding to MnSOD and inhibiting its ROS-scavenging activity (39). ROS accumulated by the upregulation of p53 eventually trigger the induction of apoptosis. However, in this study, we did not observe any significant transcriptional changes in pro- and anti-oxidant genes as a result of nutlin-3 treatment, which is consistent with the absence of an effect of PFT- $\alpha$ on p53-induced MEK1/2-ERK1/2 phosphorylation and the interaction between p53 and MnSOD (data not shown). In addition, the overexpression of MnSOD did not prevent nutlin-3-induced ROS accumulation. Therefore, it can be postulated that binding targets of p53 other than MnSOD may be present in the mitochondria that are responsible for ROS accumulation in nutlin-3-treated cells. It is noteworthy that ROS accumulation induced by nutlin-3 treatment resulted in the survival rather than the apoptosis of U2OS cells, as has been reported thus far, indicating the peculiarity of the mechanism and the role of ROS accumulation as a result of nutlin-3 treatment in this cell line.

Although MEK1/2 and ERK1/2 are independent of the transcriptional activity of p53, as evidenced by the effect of PFT- $\alpha$ on the activation of these kinases, the mechanisms for their activation appear to vary regarding the dependency on ROS. Whereas ERK1/2 activation was suppressed by the prevention of ROS induction and the mitochondrial transloca- 
tion of $\mathrm{p} 53$, this prevention had no effect on MEK1/2 activation. We were also unable to observe the dependency of MEK1/2 activation on Raf kinases (data not shown). Of note, PFT- $\mu$ augmented the nutlin-3-induced phosphorylation of MEK1/2 as well as the expression of p21WAF1 protein (Fig. 4C). PFT- $\mu$ has been reported to have no effect on the transcriptional activity of p53 (40) and, moreover, the nutlin-3-induced increase of p21 mRNA and the level of MEK1/2 protein were not affected by PFT- $\mu$-pre-treatment (Fig. 4C; data not shown), suggesting that the potentiating effect of PFT- $\mu$ on the phosphorylation of MEK1/2 and the expression of p21WAF1 protein occurred at the post-transcriptional level. In addition, on the immunoblot analysis of subcellular fractions, p53 accumulated in the cytosol of PFT- $\mu$-pre-treated cells. Therefore, it may be inferred that p53 activates MEK1/2 at the post-transcriptional level and through cytosolic events that need to be identified hereafter to elucidate the mechanism behind the p53-induced MEK1/2 activation.

Nutlin-3 is a promising anticancer drug since it does not induce genomic DNA damage that can contribute to carcinogenesis secondary to anticancer therapy. In the present study, we propose a mechanism that explains the manner by which nultin-3 treatment activates the cell survival pathway simultaneously with apoptosis via ROS accumulation and MEK1/2-ERK1/2 activation induced by the mitochondrial translocation of p53 in human cancer cells. For the optimal use of nutlin-3, interfering with the nutlin-3-induced survival pathway is critical. In order to characterize the nutlin-3induced survival pathway in detail, proteins interacting with p53 in the mitochondria, which are critical for ROS accumulation and the major determinants between ROS generation and apoptosis induced by mitochondrial p53, should be identified in the future and their distribution or expression characteristics among cancer cells, as well as their effect on nutlin-3-induced apoptosis should be elucidated for the optimal use of nutlin-3 against cancer.

\section{Acknowledgements}

We are deeply grateful to Professor Ute M. Moll at Stony Brook University and her colleagues for providing us with the Lp53WT and Lp53CBT plasmids and helpful advice. This study was supported by the Basic Science Research Program through the National Research Foundation of Korea (NRF) funded by the Ministry of Education, Science and Technology (2011-0027115).

\section{References}

1. Menendez D, Inga A and Resnick MA: The expanding universe of p53 targets. Nat Rev Cancer 9: 724-737, 2009.

2. Riley T, Sontag E, Chen P and Levine A: Transcriptional control of human p53-regulated genes. Nat Rev Mol Cell Biol 9: 402-412, 2008.

3. Honda R, Tanaka H and Yasuda H: Oncoprotein MDM2 is a ubiquitin ligase E3 for tumor suppressor p53. FEBS Lett 420 25-27, 1997.

4. Oliner JD, Pietenpol JA, Thiagalingam S, Gyuris J, Kinzler KW and Vogelstein B: Oncoprotein MDM2 conceals the activation domain of tumor suppressor p53. Nature 362: 857-860, 1993.

5. Reinhardt HC and Schumacher B: The p53 network: cellular and systemic DNA damage responses in aging and cancer. Trends Genet 28: 128-136, 2012.
6. Sill H, Olipitz W, Zebisch A, Schulz E and Wölfler A: Therapy-related myeloid neoplasms: pathobiology and clinical characteristics. Br J Pharmacol 162: 792-805, 2011.

7. Travis LB, Ng AK, Allan JM, et al: Second malignant neoplasms and cardiovascular disease following radiotherapy. J Natl Cancer Inst 104: 357-370, 2012

8. Shangary S and Wang S: Targeting the MDM2-p53 interaction for cancer therapy. Clin Cancer Res 14: 5318-5324, 2008.

9. Dickens MP, Fitzgerald R and Fischer PM: Small-molecule inhibitors of MDM2 as new anticancer therapeutics. Semin Cancer Biol 20: 10-18, 2010.

10. Vassilev LT, Vu BT, Graves B, et al: In vivo activation of the p53 pathway by small-molecule antagonists of MDM2. Science 303: 844-848, 2004.

11. Secchiero P, Bosco R, Celeghini C and Zauli G: Recent advances in the therapeutic perspectives of nutlin-3. Curr Pharm Des 17: 569-577, 2011.

12. Tovar C, Rosinski J, Filipovic Z, et al: Small-molecule MDM2 antagonists reveal aberrant p53 signaling in cancer: implications for therapy. Proc Natl Acad Sci USA 103: 1888-1893, 2006.

13. Mendrysa SM, O'Leary KA, McElwee MK, et al: Tumor suppression and normal aging in mice with constitutively high p53 activity. Genes Dev 20: 16-21, 2006.

14. Mebratu Y and Tesfaigzi Y: How ERK1/2 activation controls cell proliferation and cell death: Is subcellular localization the answer? Cell Cycle 8: 1168-1175, 2009.

15. McCubrey JA, Steelman LS, Chappell WH, et al: Roles of the Raf/MEK/ERK pathway in cell growth, malignant transformation and drug resistance. Biochim Biophys Acta 1773: 1263-1284, 2007.

16. Persons DL, Yazlovitskaya EM and Pelling JC: Effect of extracellular signal-regulated kinase on p53 accumulation in response to cisplatin. J Biol Chem 275: 35778-35785, 2000.

17. Sablina AA, Chumakov PM, Levine AJ and Kopnin BP: p53 activation in response to microtubule disruption is mediated by integrin-Erk signaling. Oncogene 20: 899-909, 2001.

18. She QB, Bode AM, Ma WY, Chen NY and Dong Z: Resveratrolinduced activation of p53 and apoptosis is mediated by extracellular-signal-regulated protein kinases and p38 kinase. Cancer Res 61: 1604-1610, 2001.

19. Lin T, Mak NK and Yang MS: MAPK regulate $\mathrm{p} 53$-dependent cell death induced by benzo[a]pyrene: involvement of $\mathrm{p} 53$ phosphorylation and acetylation. Toxicology 247: 145-153, 2008.

20. Lee SW, Fang L, Igarashi M, Ouchi T, Lu KP and Aaronson SA: Sustained activation of Ras/Raf/mitogen-activated protein kinase cascade by the tumor suppressor p53. Proc Natl Acad Sci USA 97: 8302-8305, 2000.

21. Fang L, Li G, Liu G, Lee SW and Aaronson SA: p53 induction of heparin-binding EGF-like growth factor counteracts p53 growth suppression through activation of MAPK and PI3K/Akt signaling cascades. EMBO J 20: 1931-1939, 2001.

22. Ongusaha PP, Kim JI, Fang L, et al: p53 induction and activation of DDR1 kinase counteracts p53-mediated apoptosis and influence p53 regulation through a positive feedback loop. EMBO J 22: 1289-1301, 2003.

23. Yin Y, Liu YX, Jin YJ, Hall EJ and Barrett JC: PAC1 phosphatase is a transcription target of p53 in signaling apoptosis and growth suppression. Nature 422: 527-531, 2003.

24. Ueda K, Arakawa H and Nakamura Y: Dual-specificity phosphatase 5 (DUSP5) as a direct transcriptional target of tumor suppressor p53. Oncogene 22: 5586-5591, 2003.

25. Li M, Zhou JY, Ge Y, Matherly LH and Wu GS: The phosphatase MKP1 is a transcriptional target of p53 involved in cell cycle regulation. J Biol Chem 278: 41059-41068, 2003.

26. Kojima K, Konopleva M, Samudio IJ, Ruvolo V and Andreeff M: Mitogen-activated protein kinase kinase inhibition enhances nuclear proapoptotic function of p53 in acute myelogenous leukemia cells. Cancer Res 67: 3210-3219, 2007.

27. Zhang W, Konopleva M, Burks JK, et al: Blockade of mitogenactivated protein kinase/extracellular signal-regulated kinase kinase and murine double minute synergistically induces apoptosis in acute myeloid leukemia via $\mathrm{BH} 3$-only proteins Puma and Bim. Cancer Res 70: 2424-2434, 2010.

28. Schmittgen TD and Livak KJ: Analyzing real-time PCR data by the comparative C(T) method. Nat Protoc 3: 1101-1108, 2008.

29. Jang JY, Kim MK, Jeon YK, et al: Adenovirus adenine nucleotide translocator-2 shRNA effectively induces apoptosis and enhances chemosensitivity by the down-regulation of ABCG2 in breast cancer stem-like cells. Exp Mol Med 44: 251-259, 2012. 
30. Sauer L, Gitenay D, Vo C and Baron VT: Mutant p53 initiates a feedback loop that involves Egr-1/EGF receptor/ERK in prostate cancer cells. Oncogene 29: 2628-2637, 2010.

31. McCubrey JA, Lahair MM and Franklin RA: Reactive oxygen species-induced activation of the MAP kinase signaling pathways. Antioxid Redox Signal 8: 1775-1789, 2006.

32. Liu CM, Sun YZ, Sun JM, Ma JQ and Cheng C: Protective role of quercetin against lead-induced inflammatory response in rat kidney through the ROS-mediated MAPKs and NF-KB pathway. Biochim Biophys Acta 1820: 1693-1703, 2012.

33. Liu B, Chen Y and St Clair DK: ROS and p53: a versatile partnership. Free Radic Biol Med 44: 1529-1535, 2008.

34. Sansome C, Zaika A, Marchenko ND and Moll UM: Hypoxia death stimulus induces translocation of $\mathrm{p} 53$ protein to mitochondria. Detection by immunofluorescence on whole cells. FEBS Lett 488: 110-115, 2001.

35. Mihara M, Erster S, Zaika A, et al: p53 has a direct apoptogenic role at the mitochondria. Mol Cell 11: 577-590, 2003.
36. Palacios G and Moll UM: Mitochondrially targeted wild-type p53 suppresses growth of mutant p53 lymphomas in vivo. Oncogene 25: 6133-6139, 2006

37. Talos F, Petrenko O, Mena P and Moll UM: Mitochondrially targeted p53 has tumor suppressor activities in vivo. Cancer Res 65: 9971-9981, 2005.

38. Vaseva AV, Marchenko ND and Moll UM: The transcriptionindependent mitochondrial p53 program is a major contributor to nutlin-induced apoptosis in tumor cells. Cell Cycle 8: 1711-1719, 2009.

39. Zhao Y, Chaiswing L, Velez JM, et al: p53 translocation to mitochondria precedes its nuclear translocation and targets mitochondrial oxidative defense protein-manganese superoxide dismutase. Cancer Res 65: 3745-3750, 2005.

40. Strom E, Sathe S, Komarov PG, et al: Small-molecule inhibitor of p53 binding to mitochondria protects mice from gamma radiation. Nat Chem Biol 2: 474-479, 2006. 